\title{
Denken Sie bei chronischer Diarrhö auch an eine mikroskopische Kolitis!
}

\begin{abstract}
Bei der differenzialdiagnostischen Abklärung einer chronischen Diarrhöe müssen neben einer funktionellen Störung und Medikamenten insbesondere auch chronisch entzündliche Darmerkrankungen diskutiert werden. Zu selten wird an eine mikroskopische Kolitis gedacht.
\end{abstract}

Während eine akute Diarrhö fast immer infektiös bedingt ist und nur in Risikosituationen mittels Erregernachweis weiter abgeklärt werden muss, erfordert eine länger als 3 Wochen anhaltende chronische Diarrhö eine weiterführende Diagnostik. „Doch nur bei $40 \%$ solcher Patienten lässt sich eine organische Ursache finden“, erläuterte Dr. Wolfgang Vogt, Esslingen.

\section{In circa $10 \%$ der Fälle handelt es sich um eine CED}

Am Anfang der Diagnostik sollte immer eine ausführliche Anamnese stehen. Nach einer Fernreise sei eine chronische Lamblieninfektion die häufigste Ursache einer anhaltenden Diarrhö.

Bei den Medikamenten kommen vor allem Metformin, Acarbose, Tyrosinkinaseinhibitoren und HIV-Therapeutika in Frage.

Aber auch NSAR, SSRI und ACEHemmer können der Auslöser sein. Führt dies nicht zu einem Ergebnis, so besteht die Indikation für eine Koloskopie; denn in ca. 10\% der Fälle handelt es sich um eine chronisch entzündliche Darmerkrankung.

\section{Immer Biopsien}

Eine Erkrankung, an die zu selten bei chronischer Diarrhö gedacht wird, ist die mikroskopische Kolitis. Es handelt sich um einen Entzündungsprozess unklarer Ätiologie, der nicht zu Schleimhautveränderungen führt und deshalb endoskopisch nicht erfasst werden kann.

„Deshalb sollten bei Patienten mit chronischer Diarrhö immer Biopsien entnommen werden, auch wenn die Schleimhaut makroskopisch intakt ist", so Vogt. Dies ist wichtig, da solche Patienten ansonsten mit dem Etikett „Reizdarm“ belegt werden. Mit dem topischen Steroid Budesonid (z.B. Budenofalk ${ }^{\circledR}$ ) steht für dieses Krankheitsbild aber eine sehr effektive Therapie zur Verfügung.

\section{Unklare Leberwerterhöhung? Nicht immer liegt's am Alkohol!}

Bei erhöhten Leberwerten liegt's nicht immer am Alkohol. Wenn auch eine chronische Virushepatitis oder eine medikamentöse Ursache ausgeschlossen sind, muss an eine autoimmune Hepatitis oder eine hereditäre Erkrankung gedacht werden.

Finden sich bei einem Patienten erhöhte Leberwerte, so wird zunächst an Alkohol oder eine nicht-alkoholische Fettleber gedacht. Als weiteres wird dann meist die Hepatitisserologie veranlasst. „Führt dies alles nicht zu einer Diagnose, so sollten auch seltene Ursachen diskutiert werden“, so Prof. Dr. Uta Merle, Heidelberg. Dazu gehören hereditäre metabolische Erkrankungen wie die Hämochromatose und der Morbus Wilson.

Die Hämochromatose ist eine autosomal-rezessiv vererbte Eisenspeicherkrankheit, bei der die intestinale Eisenresorption pathologisch erhöht ist. Dies führt zur Schädigung von Leber, Herz, Pankreas, Hypophyse und Gelenken. Beim Morbus Wilson ist die Kupferhomöostase gestört.

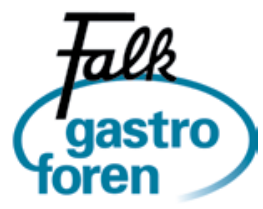

Besuchen Sie das nächste Falk Gastroforum

"Viszeralmedizin heute: von der Empirie zur personalisierten Medizin" am Samstag, den 4. Juni 2016 in Essen;

Infos: www.fortbildung@falkfoundation.de

Auch dies führt zu einer Leberschädigung bis hin zur Zirrhose.

\section{Autoantikörper und erhöhtes IgG}

$\mathrm{Zu}$ den seltenen Hepatopathien gehört auch die Autoimmunhepatitis, die überwiegend Frauen betrifft. Typische Symptome sind Abgeschlagenheit und Arthralgien. Die Diagnose gilt als gesichert, wenn entsprechende Autoantikörper und ein erhöhtes IgG nachgewiesen sind und eine virale Hepatitis ausgeschlossen ist. Die Standardtherapie ist Steroid plus Azathioprin (z. B. Azafalk ${ }^{\circledast}$ ), wobei in einer Studie das topische Budesonid einem konventionellen Steroid sogar überlegen war. Als ultima ratio kommt nur die Lebertransplantation in Betracht.

(Peter Stiefelhagen)

Seminar: XVI. Gastroenterologie-Seminarwoche Titisee der Falk Foundation e.V., 28.2.2016 